\title{
Efficient Computation of Outage Probabilities Due to Polarization Effects in a WDM System Using a Reduced Stokes Model and Importance Sampling
}

\author{
Ivan T. Lima, Jr., Student Member, IEEE, Aurenice O. Lima, Student Member, IEEE, John Zweck, and
} Curtis R. Menyuk, Fellow, IEEE

\begin{abstract}
We propose a technique that uses Monte Carlo simulations with importance sampling and a reduced Stokes model to compute the probability density function of the $Q$ factor and the outage probability for a channel in a long-haul wavelength-division-multiplexed optical-fiber transmission system due to the combination of polarization mode dispersion, polarization dependent loss, and polarization dependent gain. This technique allows us to compute outage probabilities as small as $10^{-6}$ at a fraction of the computational cost required by standard Monte Carlo simulations.
\end{abstract}

Index Terms-Fiber properties, optical communications, polarization, polarization-sensitive devices.

\section{INTRODUCTION}

A MAJOR GOAL in the design of wavelength-division-multiplexed (WDM) optical fiber transmission systems is to minimize the probability of channel outages due to the polarization effects. System designers commonly allocate a prescribed margin to polarization effects, such as $2 \mathrm{~dB}$, with a fixed probability that the margin will be exceeded, such as $10^{-6}$, corresponding to about half a minute per year. When this margin is exceeded, an outage is said to occur. Because outages are extremely rare, it has been difficult to study them theoretically using standard Monte Carlo simulations and experimentally using laboratory and field experiments.

There are three polarization effects that lead to impairment in long-haul optical fiber transmission systems: polarization-mode dispersion (PMD), polarization-dependent loss (PDL), and polarization-dependent gain (PDG). In typical trans-oceanic systems, the PMD is so small that it does not distort the pulses within a channel, since for these systems the mean of the accumulated differential-group delay of the transmission line does not exceed $10 \%$ of the bit period [1]. However, PMD does cause the polarization states of the different channels to gradually drift apart on the Poincare sphere. Because the gain saturation of the optical amplifiers keeps the total power almost constant, PMD can decrease the signal-to-noise ratios of those channels that repeatedly undergo high loss in devices with PDL, which can in

Manuscript received July 15, 2002; revised September 18, 2002. This work was supported in part by the Science Applications International Corporation, in part by the Department of Energy, and in part by the National Science Foundation. The work of I. T. Lima, Jr. was also supported by the National Research Council of Brazil (CNPq).

The authors are with the Department of Computer Science and Electrical Engineering, University of Maryland, Baltimore, MD 21250 USA (e-mail lima@engr.umbc.edu).

Digital Object Identifier 10.1109/LPT.2002.805863 turn result in channel outages. Wang and Menyuk [2] proposed the reduced Stokes model as a tool for the computation of the penalty induced by polarization effects in long-haul transmission systems. The reduced model only follows the evolution of the four Stokes parameters of the signal and of the noise in each channel due to the combined effects of PMD, PDL, PDG, amplifier spontaneous emission noise, and the gain saturation of optical amplifiers. Thus, the reduced model applies when the PMD is not so large that it distorts the pulses within a single channel. For a given fiber realization, we calculate the $Q$ factor from the signal-to-noise ratio using a simple integrate and dump receiver model [3], as in [1], [2], when PDL and PDG are present and when they are absent for a fixed level of PMD. From that, we may determine the polarization penalty $\Delta Q$ (in $\mathrm{dB}$ ) due to these effects, where $\Delta Q$ is defined as the difference between the $Q$ factor without PDL and PDG and the $Q$ factor when all the polarization effects are included. We define the outage probability due to the polarization effects to be the probability that $\Delta Q$ exceeds an allowed margin.

The reduced model decreases the computational time of simulations of the polarization effects by several orders of magnitude when compared to full time and frequency domain simulations. Even so, until now efficient computation of outage probabilities has only been carried out using numerical extrapolation with a Gaussian function [2] to estimate the tails of the probability density function (pdf) of $\Delta Q$. In this letter, we develop an importance-sampling technique [4] to resolve the tails of the pdf of $\Delta Q$ and thereby efficiently compute outage probabilities as small as $10^{-6}$. In addition, we have been able to determine the accuracy of the Gaussian extrapolation of the pdf of $\Delta Q$. We extend the analysis in [5] by taking into account the effect of PDG, discussing how the effectiveness of the importance-sampling method depends on the number of WDM channels and on the PDG, and by explaining the method in more detail. Importance sampling was first applied to optical fiber communications by Biondini et al. [6], where it was used to study the PMD-induced differential group delay in a fiber or an emulator with a limited number of sections. The method has also been applied to study the effectiveness of both electronic [7] and optical [8] PMD compensators.

\section{THEORY}

Importance sampling is a well known technique in statistics that makes more efficient use of Monte Carlo simulations to 
compute the probability of rare events. For the problem of computing the outage probability due to the polarization effects for a given channel in a WDM system, the rare events that interest us are those for which the penalty $\Delta Q$ is close to the allowed margin in a given channel. This value is large compared to the average or expected value of $\Delta Q$. To successfully apply importance sampling to this problem, we need some knowledge of which random realizations of the fiber PMD produce these large $\Delta Q$ penalties. These random fiber realizations are those for which the Stokes vector of the signal in the given channel is more likely to be aligned with the high-loss axis of the PDL elements than with the low-loss axis. We stress that the method is self validating. If we bias our samples incorrectly, then the variance of the results is large. ${ }^{1}$

The basic idea of Monte Carlo simulations with importance sampling is to bias the pdfs of the sources of randomness so that the rare events of interest occur more frequently than they would in standard Monte Carlo simulations and then to weight the samples by the likelihood that the biased random sources would actually appear in standard Monte Carlo simulations. Using importance sampling, the probability $P_{I}$ of an event defined by the indicator function $I(\mathbf{x})$, is expressed as

$$
P_{I}=\frac{1}{M} \sum_{i=1}^{M} I\left(\mathbf{x}_{i}\right) L\left(\mathbf{x}_{i}\right)
$$

where $L(\mathbf{x})=p(\mathbf{x}) / p^{*}(\mathbf{x})$ is the likelihood ratio, $p(\mathbf{x})$ and $p^{*}(\mathbf{x})$ are the unbiased and biased pdfs of a random vector $\mathbf{x}$, and $\mathbf{x}_{1}, \ldots, \mathbf{x}_{M}$ are sampled instances of $\mathbf{x}$. In our application, the random vector $\mathbf{x}$ corresponds to the random realization of the fiber PMD, and the unbiased pdf is known a priori. The indicator function $I$ is chosen to compute the probability of having $\Delta Q$ within a given range, such as a bin in a histogram.

The key issue in applying importance sampling is to choose the biased pdf $p^{*}(\mathbf{x})$. In the reduced model, the PDL is lumped in the optical amplifiers. So, to compute the outage probability for a given channel, the appropriate pdfs to bias are the pdfs of the angles $\theta_{n}$ between the polarization state of the channel and the polarization state that undergoes the highest loss due to PDL in the $n$th optical amplifier. By biasing $\cos \theta_{n}$ toward one, we increase the likelihood that the $\Delta Q$ of the channel will be large. The angles $\theta_{n}$ are directly determined by the realization of the random mode coupling in the last birefringent section of the fiber that precedes the $n$th optical amplifier. Thus, the values of $\cos \theta_{n}$ play the role of the components of the random vector $\mathbf{x}$ in (1). In standard Monte Carlo simulations in which the PMD is modeled using the coarse step method [9], the cosines of each of the angles $\theta_{n}$ are uniformly distributed in the interval $[-1,1]$. We note that an unbiased importance sampling simulation, for which $L(\mathbf{x})=1$, is exactly the same as a standard Monte Carlo simulation. However, in biased importance sampling we fix a biasing parameter $\alpha$ and select the $\cos \theta_{n}$ from a biased pdf, $p_{\alpha}^{*}(\cos \theta)$. In this letter, we used the biased pdf

$$
p_{\alpha}^{*}(\cos \theta)=\frac{\alpha}{1-e^{-2 \alpha}} e^{-\alpha(1-\cos \theta)}
$$

${ }^{1}$ There is one important caveat: If two or more disconnected regions in the phase space produce the same penalty, then it is possible in principle to have low variance and for the result to still be incorrect. This outcome does not appear to be physically possible for the problem studied here. which biases $\cos \theta$ toward 1 when $\alpha$ is positive and which corresponds to standard Monte Carlo simulations in the limit $\alpha=0$. The likelihood ratio for this pdf is given by

$$
L_{\alpha}(\cos \theta)=\frac{1-e^{-2 \alpha}}{2 \alpha} e^{\alpha(1-\cos \theta)} .
$$

Since the unbiased $\cos \theta_{n}$ are independent random variables, the likelihood ratio for a biased realization of the fiber PMD is equal to the product of the likelihood ratios for each of its biased angles. The actual pdf of $\Delta Q$ is obtained using (1) by weighting each fiber sample by its likelihood ratio, which is the likelihood that the biased fiber realization would actually occur in an unbiased simulation. By combining several Monte Carlo simulations with different values of the biasing parameter $\alpha$, including $\alpha=0$, we can statistically resolve the pdf of $\Delta Q$ in any desired range. An important issue is to determine the value of the biasing parameter $\alpha$ that enables us to statistically resolve the histogram of $\Delta Q$ over a range of large $\Delta Q$ values whose probability is on the order of a given target probability $P_{\alpha}$, such as $P_{\alpha}=10^{-6}$. We can show heuristically that the required value of $\alpha$ is the one for which the target probability $P_{\alpha}$ is equal to the likelihood ratio of the biased realization of the fiber PMD evaluated at the expected value of the random variable $\cos \theta$ with biased pdf $p_{\alpha}^{*}$. That is, $\alpha$ satisfies the equation

$$
P_{\alpha}=L_{\alpha}^{N}(E[\cos \theta])=L_{\alpha}^{N}\left(\frac{1+e^{-2 \alpha}}{1-e^{-2 \alpha}}-\frac{1}{\alpha}\right)
$$

where $N$ is the number of optical amplifiers, and $E[\cdot]$ is the expectation operator. Our motivation for this heuristic comes from (1) and from the observation that, over a given range, the biased samples statistically resolve the histogram of $\Delta Q$ when the indicator function for this range has the value 1 for a large proportion of the biased samples. For the simulation results in this letter, we chose $\alpha=0$ which produces unbiased samples, together with $\alpha=0.3$ and $\alpha=0.6$ whose target probabilities are $P_{0.3}=1.5 \times 10^{-2}$ and $P_{0.6}=1.8 \times 10^{-7}$, respectively. Just as in a standard Monte Carlo simulation, as we increase the number of samples with bias parameter $\alpha$, we increase the size of the interval about $P_{\alpha}$ for which the histogram of $\Delta Q$ is well resolved. For the results in this letter, the final histograms of the $\Delta Q$ penalty were obtained by dividing the range of penalties in decibels into 50 bins, and by combining the three biased pdfs using the stratified merging heuristic of [10], in which the probability of a bin with indicator function $I$ is obtained by applying (1) to the biased pdf that has the largest number of hits in that bin [10].

\section{NUMERICAL RESULTS}

In this section, we compute outage probabilities due to polarization effects for a trans-oceanic WDM system by applying importance sampling to resolve the tails of the pdf of $\Delta Q$. We validated our results in two steps. First, as described in [2] and [5], we validated the reduced Stokes model by comparison to a full time- and frequency-domain model based on the Manakov-PMD equation [9]. Second, by performing reduced model simulations as described in the following paragraph, we validated our method of computing outage probabilities using Monte Carlo simulations with importance sampling by making comparisons with extensive standard Monte Carlo simulations. 

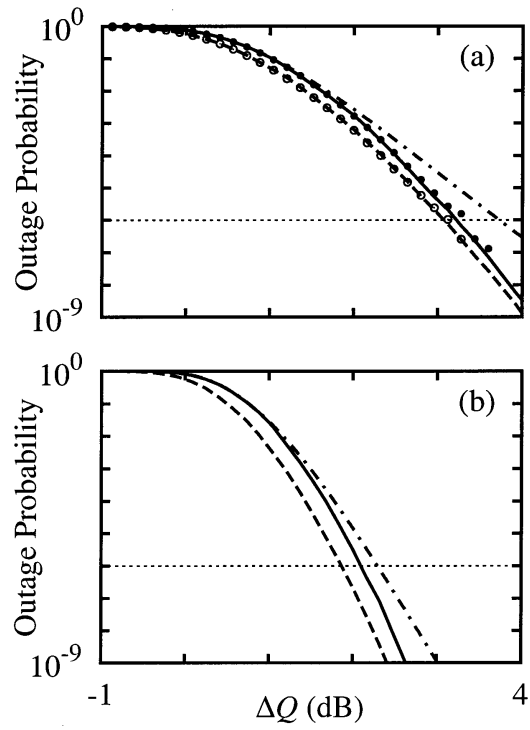

Fig. 1. Outage probability as a function of the allowed $\Delta Q$ margin for an eight-channel WDM system. The solid and dashed curves show the outage probability when the PDG is equal to 0.06 and $0 \mathrm{~dB}$, respectively, computed using $3 \times 10^{4}$ Monte Carlo simulations with importance sampling. The dotted-dashed curves in Fig. 1 show the outage probability for the system with PDG obtained from the linear-scale Gaussian pdfs that have the same means and standard deviations as the pdfs of $\Delta Q$. (a) System with PDL $=0.2 \mathrm{~dB}$ per optical amplifier. The solid circles show results that we obtained from standard Monte Carlo simulations with $7.5 \times 10^{6}$ samples when the PDG is $0.06 \mathrm{~dB}$ per amplifier, and we obtained the open circles using $1.5 \times 10^{7}$ standard Monte Carlo samples when there is no PDG. (b) System with PDL $=0.13 \mathrm{~dB}$ per optical amplifier. The horizontal dotted line in (a) and (b) shows the $10^{-6}$ probability level.

Our results are for a $10 \mathrm{~Gb} / \mathrm{s}$ return-to-zero system with eight WDM channels spaced $1 \mathrm{~nm}(124 \mathrm{GHz})$ apart. The total propagation distance was $8,910 \mathrm{~km}$ and the amplifier spacing was $33 \mathrm{~km}$. The PMD was $0.1 \mathrm{ps} / \mathrm{km}^{1 / 2}$. In Fig. 1 , we plot the outage probability as a function of the allowed $\Delta Q$ margin. In Fig. 1(a), the PDL was $0.2 \mathrm{~dB}$ per amplifier and in Fig. 1(b) the PDL was $0.13 \mathrm{~dB}$ per amplifier. The solid and dashed curves show the outage probability when the PDG is equal to 0.06 and $0 \mathrm{~dB}$, respectively, computed using $3 \times 10^{4}$ Monte Carlo simulations with importance sampling. In Fig. 1(a), we obtained the solid circles from standard Monte Carlo simulations with $7.5 \times 10^{6}$ samples when the PDG is $0.06 \mathrm{~dB}$ per amplifier, and we obtained the open circles from $1.5 \times 10^{7}$ standard Monte Carlo samples when there is no PDG. The standard Monte Carlo simulations shown in Fig. 1(a) required over two months of continuous processing in a Pentium $750-\mathrm{MHz}$ processor. The agreement in Fig. 1(a) between the results obtained using Monte Carlo simulations with importance sampling and the ones using standard Monte Carlo simulations is excellent. We used the same computer code and the same processor for the two methods; the only difference is that we did not bias the fiber birefringence for the standard Monte Carlo method. The dotted-dashed curves in Fig. 1 show the outage probability for the system with PDG obtained from the linear-scale Gaussian pdfs that have the same means and standard deviations as the pdfs of $\Delta Q$. Even though the pdf of $\Delta Q$ is very close to a Gaussian pdf near its mean value, the Gaussian pdf overestimates the $\Delta Q$ margin at an outage probability of $10^{-6}$ by about $0.6 \mathrm{~dB}$ when the PDL is $0.2 \mathrm{~dB}$ per amplifier.

\section{CONCLUSION}

In conclusion, we have demonstrated that it is possible to use Monte Carlo simulations with importance sampling to efficiently and accurately calculate outage probabilities on the order of $10^{-6}$ due to the combination of the polarization effects of PMD, PDL, and PDG using a reduced Stokes model, provided that the PMD is small enough that it does not distort the pulses within a channel. By using importance sampling, we can reduce the time required to compute small outage probabilities by three orders of magnitude when compared with standard Monte Carlo simulations. This result holds independent of the particular choice of receiver model. We are currently improving our receiver model to include realistic filter shapes [11] and to account for noise repolarization during transmission.

\section{ACKNOWLEDGMENT}

The authors thank W. L. Kath and G. Biondini for many valuable discussions.

\section{REFERENCES}

[1] E. Golovchenko, "The challenges of designing long-haul WDM systems," in Tutorial Sessions OFC 2002, 2002, Paper TuL, pp. 1-52.

[2] D. Wang and C. R. Menyuk, "Calculation of penalties due to polarization effects in a long-haul WDM system using a Stokes parameter model," J. Lightwave Technol., vol. 19, pp. 487-494, Apr. 2001.

[3] D. Marcuse, "Derivation of analytical expressions for the bit-error probability in lightwave systems with optical amplifiers," J. Lightwave Technol., vol. 8, pp. 1816-1823, Dec. 1990.

[4] R. Y. Rubinstein, Simulation and the Monte Carlo Method. New York: Wiley, 1981.

[5] I. T. Lima Jr., A. O. Lima, Y. Sun, J. Zweck, B. S. Marks, G. M. Carter, and C. R. Menyuk, "Computation of the outage probability due to the polarization effects using importance sampling," in Proc. OFC 2002, 2002, Paper TuI7, pp. 56-57.

[6] G. Biondini, W. L. Kath, and C. R. Menyuk, "Importance sampling for polarization-mode dispersion," IEEE Photon. Technol. Lett., vol. 14, pp. 310-312, Mar. 2002.

[7] A. O. Lima, I. T. Lima Jr., T. Adali, and C. R. Menyuk, "A novel polarization diversity receiver for PMD mitigation," IEEE Photon. Technol. Lett., vol. 14, pp. 465-467, Apr. 2002.

[8] I. T. Lima Jr., G. Biondini, B. S. Marks, W. L. Kath, and C. R. Menyuk, "Analysis of PMD compensators with fixed DGD using importance sampling," IEEE Photon. Technol. Lett., vol. 14, pp. 627-629, May 2002.

[9] D. Marcuse, C. R. Menyuk, and P. K. A. Wai, "Application of the Manakov-PMD equation to studies of signal propagation in optical fibers with randomly varying birefringence," J. Lightwave Technol., vol. 15, pp. 1735-1746, Sept. 1997.

[10] E. Veach, "Robust Monte Carlo Methods for Light Transport Simulation," Ph.D. dissertation, Stanford University, Palo Alto, CA, 1997, [Online] Available: http://graphics.stanford.edu/papers/veach_thesis/.

[11] P. J. Winzer, M. Pfennigbauer, M. M. Strasser, and W. R. Leeb, "Optimum filter bandwidth for optically preamplified NRZ receivers," $J$. Lightwave Technol., vol. 19, pp. 1263-1273, Sept. 2001. 